\title{
ON THE UPPER AND LOWER ESTIMATES OF NORMS IN VARIABLE EXPONENT SPACES
}

\author{
Tengiz Kopaliani, Nino Samashvili and Shalva ZviadadZE
}

Abstract. In the present paper we investigate some geometrical properties of the norms in Banach function spaces. Particularly there is shown that if exponent $1 / p(\cdot)$ belongs to $B L O^{1 / \log }$ then for the norm of corresponding variable exponent Lebesgue space we have the following lower estimate

$$
\left\|\sum \chi_{Q}\right\| f \chi_{Q}\left\|_{p(\cdot)} /\right\| \chi_{Q}\left\|_{p(\cdot)}\right\|_{p(\cdot)} \leqslant C\|f\|_{p(\cdot)}
$$

where $\{Q\}$ defines disjoint partition of $[0 ; 1]$. Also we have constructed variable exponent Lebesgue space with above property which does not possess following upper estimation

$$
\|f\|_{p(\cdot)} \leqslant C\left\|\sum \chi_{Q}\right\| f \chi_{Q}\left\|_{p(\cdot)} /\right\| \chi_{Q}\left\|_{p(\cdot)}\right\|_{p(\cdot)} .
$$

Mathematics subject classification (2010): 42B35, 42B20, 46B45, 42B25.

Keywords and phrases: Upper $p$-estimate, lower $q$-estimate, variable exponent Lebesgue space, HardyLittlewood maximal operator.

\section{REFERENCES}

[1] C. Bennet And R. Sharpley, Interpolation of operators, Pure Appl. Math. 129, Academic Press, 1988.

[2] E. BereZhnoi, Sharp estimates for operators on cones in ideal spaces, Trudy Mat. Inst. Steklov. 204 (1993), 3-36 (in Russian).

[3] E. BEREZHNOI, Two-weighted estimations for the Hardy-Littlewood maximal function in ideal Bannach spaces, Proc. Amer. Math. Soc. 127 (1999), 79-87.

[4] D. CruZ-Uribe, A. Fiorenza, Variable Lebesgue Spaces: Foundations and Harmonic Analysis, Birkhäuser, Basel (2013).

[5] L. Diening, Maximal function on generalized Lebesgue spaces $L^{p(\cdot)}$, Math. Inequal. Appl. 7 (2004), 245-253.

[6] L. Diening, Maximal function on Orlicz-Musielak spaces and generalized Lebesgue spaces, Bull. Sci. Math., (129), (2005), 657-700.

[7] L. Diening, P. HÄStÖ, P. HARJulehto And M. RŮŽIČKA, Lebesgue and Sobolev spaces with variable exponents, Springer Lecture Notes, vol. 2017, Springer-Verlag, Berlin 2011.

[8] T. Figiel, W. Johnson, A uniformly convex Banach space which contains no $l_{p}$, Compositio Mathematica 29. 2 (1974), 179-190.

[9] F. L. HERnANDEZ, C. RUIZ, Averaging and orthogonal operators on variable exponent spaces $L^{p(\cdot)}(\Omega)$, J. Math. Anal. Appl. 413 (2014), 139-153.

[10] E. Kapanadze, T. Kopaliani, A note on maximal operator on $L^{p(\cdot)}(\Omega)$ spaces, Georgian Math. J. 16, no. 2, (2008), 307-316.

[11] T. Kopaliani, On some structural properties of Banach function spaces and boundedness of certain integral operators, Czechoslovak Math. J., 54, (2004), 791-805.

[12] T. Kopaliani, Infimal convolution and Muckenhoupt $A_{p(\cdot)}$ condition in variable $L^{p}$ spaces, Arch. Math. (Basel), 89, (2007), 185-192.

[13] T. Kopaliani, A characterization of some weighted norm inequalities for maximal operators, Z. Anal. Anwend. 29 (2010), no. 4, 401-412. 
[14] A. KorenovsKII, Mean Oscillations and Equimeasurable Rearrangements of Functions, Springer, 2007.

[15] A. LERNER, Some remarks on the Hardy-Littlewood maximal function on variable Lp spaces, Math. Z., (251), (2005), 509-521.

[16] J. Lindenstrauss ANd L. TZAFriri, Classical Banach spaces, I, II, Springer-Verlag, 1977, 1979.

[17] T. SHIMOGAKI, Exponents of norms in semi-ordered line ar spaces, Bull. Acad. Polon. Sci. 13, (1965), $135-140$.

[18] S. SPAnNe, Some function spaces defined using the mean oscillation over cubes, Ann. Sc. Norm. Super. Pisa, Sci. Fis. Mat. III. 19, (1965), 593-608. 